\title{
Numerical solution of Hele-Shaw flows driven by a quadrupole
}

\author{
E. D. KELLY and E. J. HINCH \\ DAMTP, University of Cambridge, Silver Street, Cambridge CB3 9EW, UK
}

(Received 19 October 1995; in revised form 29 July 1996)

\begin{abstract}
A blob of viscous Newtonian fluid is surrounded by inviscid fluid and sandwiched in the narrow gap between two plane parallel surfaces, so that initially its plan view occupies a simply connected domain. Recently Entov, Etingoff \& Kleinbock (1993) produced some steady-state solutions for the blob placed in a quadrupole driven flow, and including the effects of surface tension. Here a numerical solution of the time-dependent problem using a Boundary Integral algorithm finds that for low values of the flow rate there exist two solutions. We find that one, which is close in shape to a circle, is stable, while the other, more deformed equilibrium, is unstable. The analysis also reveals that for certain flow strengths stable non-convex shapes also exist. If the flow strength is too large no stable equilibrium is possible.
\end{abstract}

\section{Introduction}

First considered by Hele-Shaw (1898) in his investigation of the transition to turbulence in a pipe, a Hele-Shaw cell is two plane parallel surfaces with a thin layer of fluid sandwiched in the narrow gap between them, such that $h / r \ll 1$, where $r$ is the radial dimension of the cell, and $h$ the gap width. Hele-Shaw cells provide us with an easily accessible model of such physical phenomena as two-dimensional porous media flow, and the manufacturing process of injection moulding. In the former case, by the use of sinks and sources, we may model the behaviour of flows in porous media in and around oil wells. The governing equations are directly analogous to the Darcy model (Darcy, 1856) which has been widely used in soil mechanics for many varieties of porous media. Recent interest in this analogy may be dated to Polubarinova-Kochina (1945). The initial motivation for this research stems from problems encountered with a particular technique of secondary oil recovery. This method involves the pumping of water through the porous rock in an oilfield, with the aim of driving out residual oil left after initial extraction techniques have been exhausted. Unfortunately, it is observed that a considerable amount of oil is left in the ground when water appears at the producing wells. This result is believed to be in part due to a fingering instability of the oil-water interface analogous to the Saffman-Taylor (1958) instability. In the case of injection moulding we may be studying the forcing of molten polymer into a mould of a certain shape which is then let solidify. The Hele-Shaw model would be useful when considering the production of plane lamina. Here a source term would model the effect of injecting the molten plastic at a point in one of the faces, a technique frequently used to help reduce the high pressures needed in the forcing as much as possible. To fill the mould completely, all the initial air must be allowed to escape, which must be done 
through air vents in regions which are the last to be filled. The Hele-Shaw model may be used to predict the location of such regions, although in the initial analysis certain assumptions and simplifications would have to be made, such as ignoring the fact that the high viscosity of the melt will give rise to significant heat generation, ignoring the associated thermal effects, and ignoring the non-Newtonian nature of the melt itself.

As mentioned above, another reason for study of this geometry is the fact that if any fluid mechanics problem is likely to be accessible to theory and to direct comparison of theory, simulation and experiment it should be this one. The flow is almost twodimensional potential flow and the basic partial differential equation is Laplace's equation. Despite this apparent simplicity, the problem has not been fully elucidated to this day, the nonlinearity of the free-surface being the basic difficulty in the prediction of the motion of the interface between the viscous fluid and the air.

Despite the assumptions necessary for the study of molten plastics mentioned above, and the fact that in actual oil reservoir conditions flow is three dimensional and may be subject to 'dispersion' of the interface, which we ignore, our motivation for studying this problem is the belief that an investigation of fluid flow in a Hele-Shaw cell will be helpful to the understanding of these problems.

One additional motivation for studying this problem is the long list of other apparently similar problems, such as directional solidification, dendritic formulation (Langer, 1980), electrodeposition, the deformation of ionospheric plasma clouds (Overman et al., 1983) and dielectric breakdown. The Hele-Shaw fingering mechanism is also thought to be relevant by analogy to the form and growth of fingered sheet intrusions in geological formations (Pollard et al., 1975). Ideas that are applicable to Hele-Shaw cells are very likely also to increase our understanding of the mathematics and physics of these other problems.

Recently non-trivial exact solutions to the Saffman-Taylor problem with surface tension were found by Kadanoff (1990) and Vasconcelos and Kadanoff (1991). Later Entov et al. (1993) produced analytic solutions for the steady shape of the interface in the presence of surface tension under the action of a multipole at the origin or infinity. The solutions of Entov et al. are time-independent, giving the steady solution for each particular geometry. Here we seek to develop a numerical, time-dependent solution for the evolution of the viscous-inviscid interface in their problem, matching the analytic steady solutions to the long-time results produced by the numerics. Previously vortex sheet methods employing the Birkhoff-Rott integral have been used in the analysis of similar problems. Tryggvason \& Aref (1983), DeGregoria \& Schwartz (1986) and Meiburg \& Homsy (1988) looked at the Saffman-Taylor fingering instability employing these methods. Dai \& Shelley (1993) also used them to investigate the effect of an expanding bubble of inviscid fluid for both the zero and small surface tension cases, an approach similar to that used in the study of vortex sheets in two-dimensional Euler flows. Here we choose to apply the similar boundary integral method in which the interface is represented by boundary elements (see Pozrikidis, 1992).

\section{Formulation}

Consider the slow motion of an incompressible Newtonian fluid in the narrow gap. Away from the multipole and the free boundary we can use the standard theory of the Hele- 
Shaw cell, as detailed in Lamb (1932). For a sufficiently small gap compared with the radial dimensions, the slow flow equations may be averaged across it to give the fluid velocity in terms of the pressure by

$$
\boldsymbol{u}=-\frac{h^{2}}{12 \mu} \nabla p,
$$

where $\boldsymbol{u}$ is the velocity of the fluid in the $x, y$-plane, $p$ is the pressure and $\mu$ is the viscosity.

As the fluid is incompressible, we also have

$$
\nabla^{2} p=0+\text { forcing, }
$$

where the forcing term is that due to the action of the multipole. Applying Green's second identity, and letting the point of observation $x$ go to the boundary, we obtain

$$
\frac{1}{2} p(\boldsymbol{x})=\text { forcing }+\oint\left(p\left(\boldsymbol{x}^{\prime}\right) \frac{\partial G\left(\boldsymbol{x}, \boldsymbol{x}^{\prime}\right)}{\partial n^{\prime}}-G\left(\boldsymbol{x}, \boldsymbol{x}^{\prime}\right) \frac{\partial p\left(\boldsymbol{x}^{\prime}\right)}{\partial n^{\prime}}\right) d l^{\prime},
$$

where $G\left(\boldsymbol{x}, \boldsymbol{x}^{\prime}\right)=1 / 2 \pi \ln \left|\boldsymbol{x}-\boldsymbol{x}^{\prime}\right|$.

The boundary of the fluid domain moves with velocity

$$
\boldsymbol{u}(\boldsymbol{x})=-\frac{h^{2}}{12 \mu} \frac{\partial p(\boldsymbol{x})}{\partial n} \boldsymbol{n} .
$$

At the interface we have a pressure jump due to surface tension, such that

$$
p(\boldsymbol{x})=\gamma \kappa(\boldsymbol{x}),
$$

where $\gamma$ is the surface tension coefficient (assumed constant), and $\kappa(x)$ is the local curvature.

Note that equation (2.5) assumes that the problem is truly a $2 \mathrm{D}$ problem. In three dimensions the curvature in the direction perpendicular to the cell must be considered. For a perfectly wetting fluid, this produces a factor of $\pi / 4$ (known as the Park and Homsy factor) multiplying the local curvature. This is beyond the scope of this paper, but details may be found in Park \& Homsy (1984).

The timescale of the problem may be derived from equation (2.1). If we consider a blob of lengthscale $D$ in the $x, y$-plane, equation (2.5) gives us that $p \sim \gamma / D$, and thus we have

$$
\boldsymbol{u} \sim \frac{h^{2}}{\mu} \frac{1}{D} \frac{\gamma}{D}
$$

If we define our timescale $T$ by $T \sim D /|\boldsymbol{u}|$, then we have

$$
T \sim \frac{D^{3}}{h^{2}} \frac{\mu}{\gamma}
$$

\section{Multipoles}

Analytic steady solutions with surface tension have been found by Entov et al. where the forcing term of equation (2.3) is a multipole (i.e. dipole, quadrupole, etc.) which produces no net volume change in the fluid.

If we consider the $x, y$-plane as the complex plane, the vector $\boldsymbol{x}$ being represented by the complex number $z=x+i y$, then the 'forcing' term of equation (2.3) is given by a 


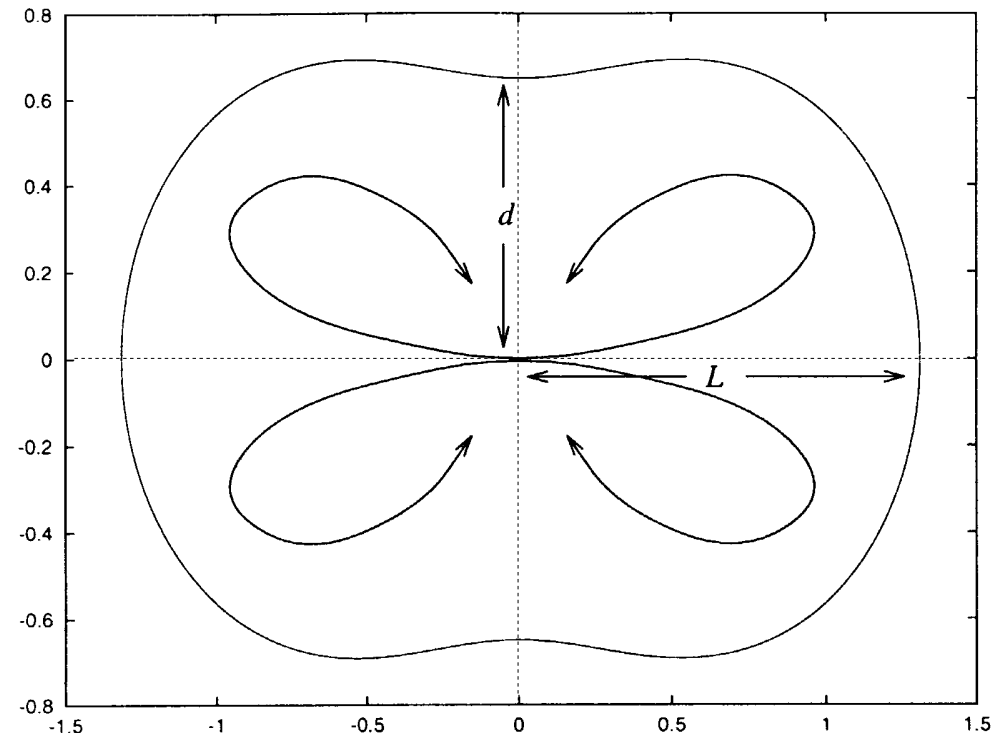

FIGURE 1. Flow generated by a quadrupole at the origin.

multipole of the form

$$
\mathfrak{R}\left(\frac{Q}{2 \pi} z^{n}\right),
$$

where $Q$ is the multipole strength, $n \in \mathbb{Z} \backslash\{0\}$ the order of the multipole and $\mathfrak{R}$ denotes the real part of the expression. For $n>0$ the geometry under consideration is that of a bubble of air surrounded by an infinite expanse of viscous fluid with the multipole at infinity. For $n<0$, the multipole is situated at the origin, within the finite expanse of the viscous fluid, which is itself surrounded by air.

The particular case of a blob of viscous fluid centred on a quadrupole $(n=-2)$ at the origin was chosen for analysis. The flow field, as depicted in Fig. 1, has no net volume change. Thus, incorporating the forcing term as given by equation (3.1), equation (2.3) becomes

$$
\frac{1}{2} p(\boldsymbol{x})=\frac{Q}{2 \pi} \frac{\left(x^{2}-y^{2}\right)}{\left(x^{2}-y^{2}\right)^{2}+4 x^{2} y^{2}}+\oint\left(p\left(\boldsymbol{x}^{\prime}\right) \frac{\partial G\left(\boldsymbol{x}, \boldsymbol{x}^{\prime}\right)}{\partial n^{\prime}}-G\left(\boldsymbol{x}, \boldsymbol{x}^{\prime}\right) \frac{\partial p\left(\boldsymbol{x}^{\prime}\right)}{\partial n^{\prime}}\right) d l^{\prime},
$$

where $\boldsymbol{x}=(x, y)$.

This gives us an inhomogeneous Fredholm equation of the first kind to be solved at each timestep. The details of the numerical techniques involved are discussed later.

\section{The deformation ratio and curve}

It will be shown here by dimensional analysis that the steady shape of the blob of fluid is determined solely by the ratio between the strength of the quadrupole, the magnitude of the surface tension, and the size of the blob. Within the viscous fluid there are two 


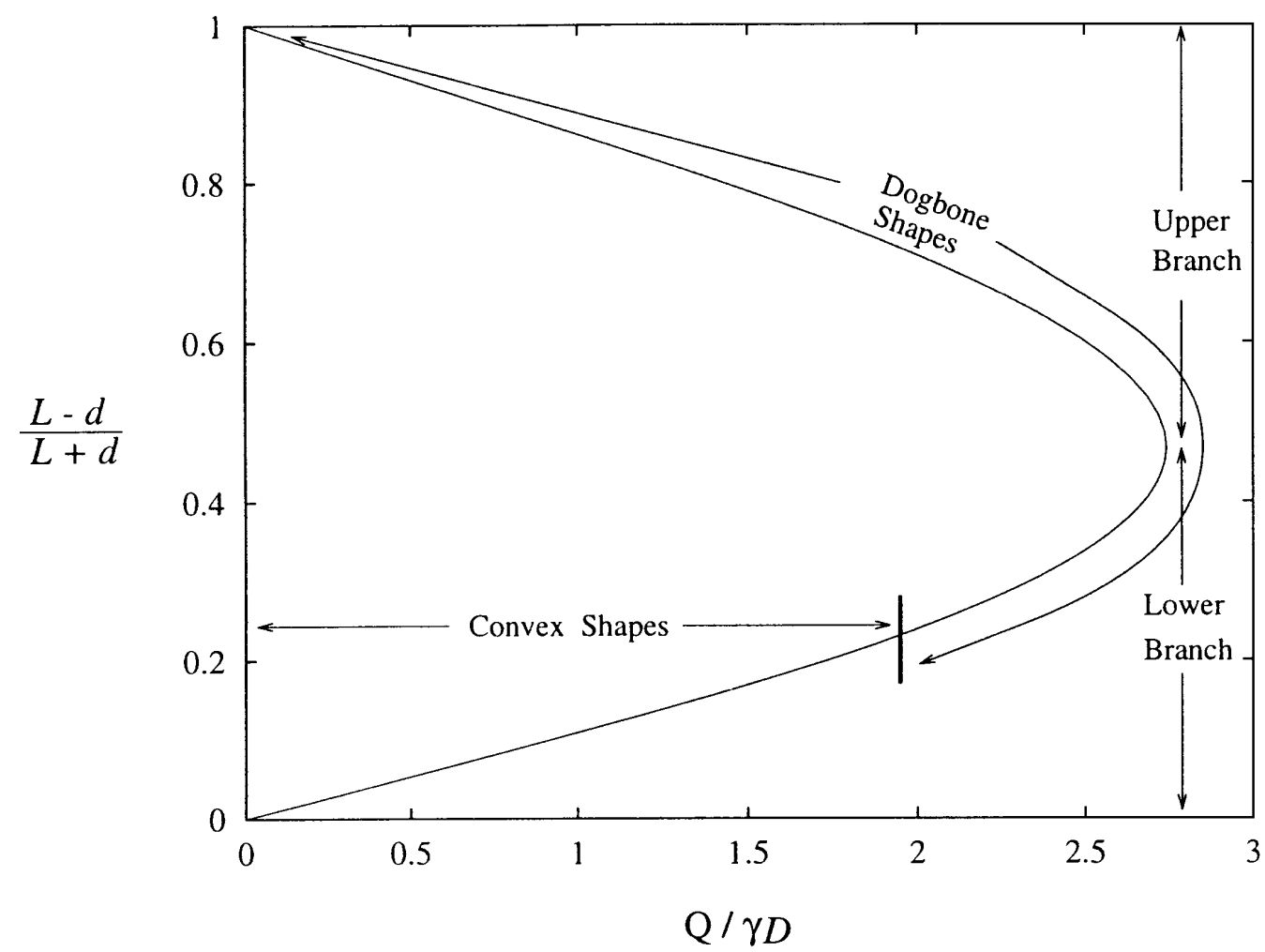

FIGURE 2. Deformation curve. $L$ and $d$ as depicted in Fig. 1.

competing effects. Consider a circular blob of radius $D$. We have from equation (3.2),

$$
p=O\left(\frac{Q}{D^{2}}\right)+O\left(\frac{\gamma}{D}\right) .
$$

Applying Darcy's law, equation (2.4), to the first term on the right-hand side gives us

$$
\boldsymbol{u}^{\text {flow }}=-\frac{h^{2}}{12 \mu} \frac{Q}{D^{3}},
$$

the velocity due to the action of the multipole, and similarly, the second term on the right-hand side produces

$$
\boldsymbol{u}^{\mathrm{ST}}=-\frac{h^{2}}{12 \mu} \frac{\gamma}{D^{2}},
$$

the velocity due to surface tension.

When the blob is in its steady shape, these two must be in equilibrium, the natural tendency of the surface tension forces being to minimise the perimeter of the blob, whereas the action of the quadrupole tends to elongate it. Balancing these two flows gives

$$
\frac{\boldsymbol{u}^{\text {flow }}}{\boldsymbol{u}^{\mathrm{ST}}}=\frac{Q}{D \gamma} .
$$

To be more precise, we now define the lengthscale of the blob $D$ to be such that the area of the blob, which is constant in time, to be $\pi D^{2}$. 


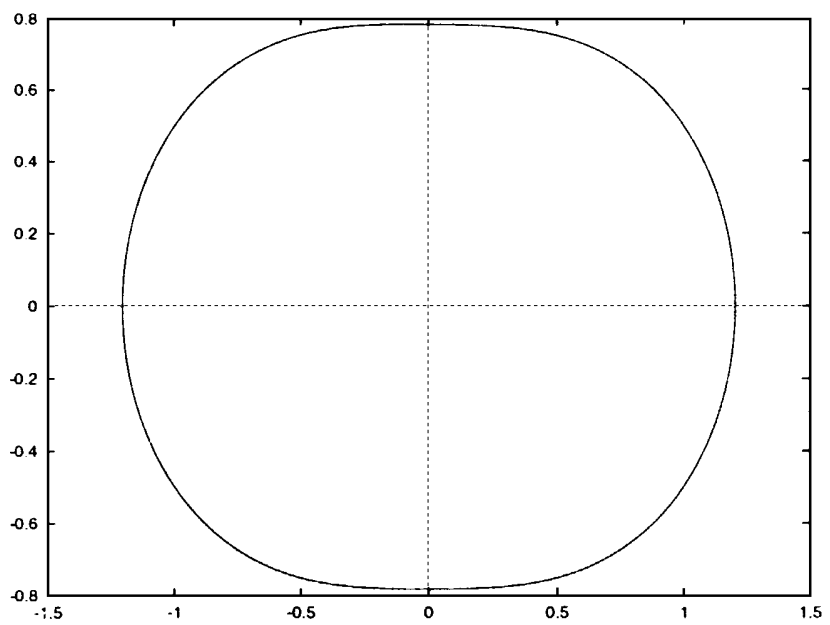

(a) Lower Branch

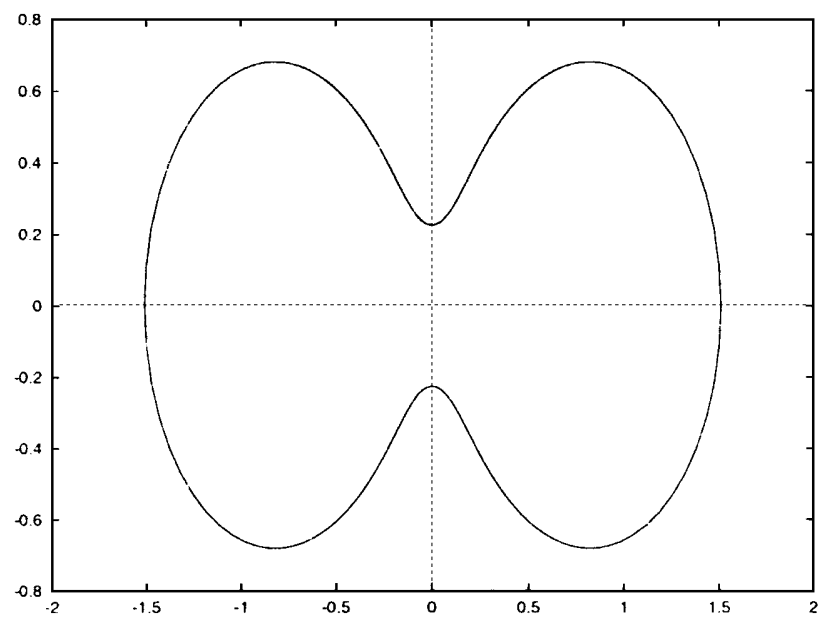

(b) Upper Branch

Figure 3. Typical steady shapes. $Q / \gamma D=1.8$. (a) $L-d / L+d=0.209$. (b) $L-d / L+d=0.743$.

Therefore the steady shape is determined solely by the ratio $Q / \gamma D$, the relative flow strength. Plotting the deformation ratio of the steady shapes, $(L-d) /(L+d)$, as predicted by Entov et al., against the flow strength results in a deformation curve, as depicted in Fig. 2. Here $( \pm L, 0)$ are the points where the interface cuts the $x$-axis, and $(0, \pm d)$ are the points where the interface cuts the $y$-axis (see Fig. 1). This relationship between the flow strength and the deformation ratio as graphed in Fig. 2, however, is not obvious from the mapping of Entov et al.. Much analysis and manipulation of their expressions was required to produce the final deformation curve.

Entov et al. (1993) noted that two deformation ratios, and consequently two steady solutions, are predicted for a range of flow strengths, and no solutions are given for values above a certain critical ratio $\left(Q / \gamma D_{\text {crit. }} \approx 2.742\right)$. It will be shown later that, as 


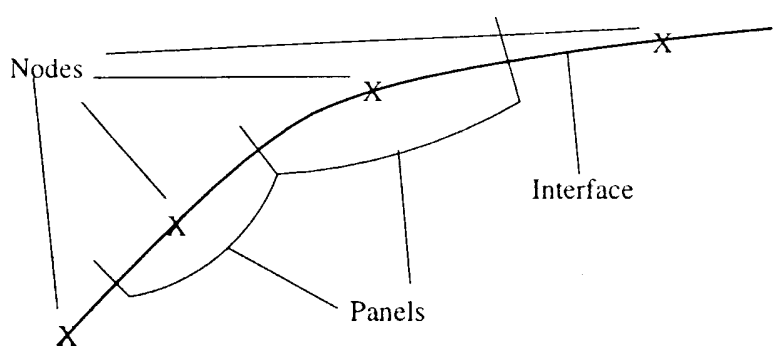

FIgURE 4. Typical interface section with nodes and panels illustrated.

might be expected, for values above this critical ratio, the surface tension forces are insufficient to balance out the quadrupole forcing, the blob continues to deform, never reaching equilibrium, and eventually splits by pinching off along the $y$-axis. At this stage the numerical code breaks down also. In fact no continuation is possible, as the interface has reached the origin, the point of action of the quadrupole. For values of $Q / \gamma D$ lying between zero and the critical value we have two solutions, with typical steady shapes being displayed in Fig. 3 for a set flow strength.

As is illustrated in Fig. 3, both convex and non-convex, or 'dogbone' as we choose also to refer to them, steady shapes exist. All shapes with deformation ratios lying on the upper branch are non-convex, and similar in shape to Fig. 3(b), with the two halves being closer and closer to pinching off along the $y$-axis as the flow strength is lowered. On the lower branch, shapes with flow strengths $\gtrsim 1.933$ are also non-convex, becoming convex below this value. It will be shown later that those shapes with deformation ratios lying on the lower branch are stable, whereas those on the upper branch are unstable to small perturbations.

\section{Numerical method}

The numerical method uses $N$ points $\boldsymbol{x}_{i}=\left(x_{i}, y_{i}\right), i=1, \ldots, N$, initially evenly spaced, on the viscous-inviscid interface. To calculate the tangential and also normal direction to the curve, a quartic spline in an arc-length-like parameter is fitted through these points. A quartic spline was chosen as a cubic proved of insufficient accuracy for the calculation of the curvature. To avoid a coordinate disaster, the $x$ and $y$ coordinates were interpolated independently, in each case the spline parameter $\tau$ having unit range between neighbouring points, e.g. $\boldsymbol{x}_{1} \equiv \boldsymbol{x}(\tau=1), \boldsymbol{x}_{2} \equiv \boldsymbol{x}(\tau=2)$, etc.

The curvature $\kappa$ at a point $\boldsymbol{x}$ is then given by

$$
\kappa=\frac{x_{\tau} y_{\tau \tau}-y_{\tau} x_{\tau \tau}}{\left[x_{\tau}^{2}+y_{\tau}^{2}\right]^{3 / 2}},
$$

and the outward normal $\boldsymbol{n}$ as

$$
\boldsymbol{n}=\frac{\left(y_{\tau},-x_{\tau}\right)}{\sqrt{ }\left(x_{\tau}^{2}+y_{\tau}^{2}\right)} .
$$

Testing on a 2:1 ellipse, the RMS error of the curvature at the nodes decreased like $N^{-4}$ over the range of interest. The RMS error in the normal vector at the nodes was $O\left(10^{-14}\right)$ over the range of interest, $40 \leqslant N \leqslant 80$. 
In order to facilitate the discretisation of the system, it is assumed that $\partial p / \partial n$ is constant at its nodal value $\partial p\left(\boldsymbol{x}_{j}\right) / \partial n$ in an interval or panel stretching between the mid-points of the spline connecting neighbouring nodes. This converts equation (3.2) into a linear system of simultaneous equations for the nodal values.

$$
E_{i j} \frac{\partial p\left(\boldsymbol{x}_{j}\right)}{\partial n}=P_{i} \quad i, j=1 \ldots N,
$$

where

$$
E_{i j}=-\int_{j_{\mathrm{thpanel}}} G\left(\boldsymbol{x}_{i}, \boldsymbol{x}^{\prime}\right) d l^{\prime}
$$

and

$$
P_{i}=\frac{1}{2} p\left(\boldsymbol{x}_{i}\right)-\frac{Q}{2 \pi} \frac{\left(x_{i}{ }^{2}-y_{i}{ }^{2}\right)}{\left(x_{i}{ }^{2}-y_{i}{ }^{2}\right)^{2}+4 x_{i}{ }^{2} y_{i}{ }^{2}}-\oint\left(p\left(\boldsymbol{x}^{\prime}\right) \frac{\partial G\left(\boldsymbol{x}_{i}, \boldsymbol{x}^{\prime}\right)}{\partial n^{\prime}}\right) d l^{\prime} .
$$

Solution of these equations gives us the unknowns $\partial p\left(\boldsymbol{x}_{j}\right) / \partial n$.

Calculation of the terms $E_{i i}$ and $P_{i}$ presented difficulties due the presence of the singularity at the centre of the $i$ th panel. This was overcome by splitting the panel containing the pole (or self-panel) in two at the pole, and performing open Romberg integration over the two halves with a tolerance of $10^{-5}$. Six-point Gaussian quadrature was used for all other $E_{i j}$ terms, $i \neq j$. Extended trapezoidal integration was used for panels other than the self-panel in the calculation of $P_{i}$, with a tolerance of $10^{-5}$.

The matrix $\boldsymbol{E}$ is, however, singular. We know theoretically that if the pressure is constant on the boundary, and the action of the quadrupole switched off, we should have no flow, i.e. $\partial p\left(\boldsymbol{x}_{j}\right) / \partial n=0 \forall j$. To purge the singular $\boldsymbol{E}$ of its zero eigensolution, we augment $\boldsymbol{E}$ to give $\boldsymbol{E}^{\prime}$ :

$$
\boldsymbol{E}^{\prime}=\boldsymbol{E}+\alpha \boldsymbol{e} \boldsymbol{e}^{\dagger},
$$

where $\boldsymbol{e}$ is the singular eigenvector and $\boldsymbol{e}^{\dagger}$ its adjoint, and where $\alpha$ is a convenient non-zero number. For this geometry, $\boldsymbol{e}$ is simply the vector of panel elements, i.e.

$$
\boldsymbol{e}^{\mathrm{T}}=\left(\delta l_{1}, \delta l_{2}, \ldots, \delta l_{N}\right),
$$

where

$$
\delta l_{i}=\int_{\boldsymbol{x}_{i-1 / 2}}^{\boldsymbol{x}_{i+1 / 2}} d l,
$$

and $\boldsymbol{x}_{i \pm 1 / 2}$ denotes the point half-way between $\boldsymbol{x}_{i}$ and $\boldsymbol{x}_{i \pm 1}$ respectively. The adjoint $\boldsymbol{e}^{\dagger}$, in this case, is the constant vector $(1,1, \ldots, 1)$.

Therefore, substituting $\boldsymbol{E}^{\prime}$ for $\boldsymbol{E}$ in equation (5.6) then gives us

$$
E_{i j}^{\prime} \frac{\partial p\left(\boldsymbol{x}_{j}\right)}{\partial n}=P_{i} \quad i, j=1 \ldots N .
$$

The matrix $\boldsymbol{E}^{\prime}$ is not singular, and therefore can be safely inverted. $L U$ decomposition was used, and no use was made of an iterative method which could take advantage of the known approximate solution from the previous timestep. To ensure that volume was conserved, the solution of equation (5.9) was also purged via

$$
\frac{\partial p}{\partial n} \rightarrow \frac{\partial p}{\partial n}-\frac{\overline{\partial p}}{\partial n},
$$


Table 1. Results showing convergence to the solution of Entov et al. for $Q / \gamma D=1.667$.

\begin{tabular}{lll}
\hline \hline$N$ & RMS error \\
\hline 40 & $4.50 \times 10^{-5}$ \\
60 & $1.88 \times 10^{-5}$ \\
80 & $1.39 \times 10^{-5}$ \\
\hline
\end{tabular}

i.e.

$$
\frac{\partial p\left(\boldsymbol{x}_{i}\right)}{\partial n} \rightarrow \frac{\partial p\left(\boldsymbol{x}_{i}\right)}{\partial n}-\oint \frac{\partial p(\boldsymbol{x})}{\partial n} d l / \oint d l \quad i=1 \ldots N .
$$

The blob area was not renormalised at any stage due to the negligible area loss observed at equilibrium.

Testing of the Laplace solver was done as follows. The forcing was turned off and the pressure set equal to the harmonic function $r^{2} \cos 2 \theta$ on the boundary of a 2:1 ellipse. The computed values for $\partial p / \partial n$ were within $1 \%$ of the known theoretical values using 40 points, and the error decreased like $N^{-2}$.

Timestepping was performed with an explicit mid-point rule. Testing of this method found the error to decrease as $\delta t^{2}$.

So far, motion of the boundary nodes is governed solely by equation (2.4), i.e. all motion is normal to the interface. However, it is useful to introduce a tangential motion also, to avoid depopulation/overcrowding of sections of the interface due to the normal motion of the nodes, which could lead to a loss of accuracy/numerical instabilities, or to concentrate nodes in areas of particular interest. Here we attempt to keep the nodes equally spaced on the interface by attaching each in turn to its two neighbours with pseudo-springs of equal strength, which at each timestep nudge the set of nodes by a small amount towards an equally-spaced equilibrium. This tangential motion is achieved via:

$$
x \rightarrow \boldsymbol{x}+\beta f(\boldsymbol{x}) \boldsymbol{t}
$$

where $\boldsymbol{t}$ is along the spline, $\beta$ a small positive number and

$$
f\left(\boldsymbol{x}_{i}\right)=\left\{\left|\boldsymbol{x}_{i+1}-\boldsymbol{x}_{i}\right|-\left|\boldsymbol{x}_{i-1}-\boldsymbol{x}_{i}\right|\right\} \quad i=1, \ldots, N
$$

where $\boldsymbol{x}_{N+1} \equiv \boldsymbol{x}_{1}$ and $\boldsymbol{x}_{0} \equiv \boldsymbol{x}_{N}$. All tangential redistribution is done simultaneously, and it is only afterwards that the velocities for the new node positions are calculated.

\section{Results}

The convergence of the time-dependent numerical solution to the analytic solution of Entov et al. was tested. Initially 40 boundary points were evenly distributed in a circle about the origin of unit radius. The flow was switched on, without ramping, with $Q / \gamma D=1.667, \delta t=5 \times 10^{-2}$, and $\beta=1.25 \times 10^{-3}$, as an initial test case. By $t=22.5$, the velocities of the points on the $y$-axis had dropped to less than $1 / 2000$ th of their initial velocities, and equilibrium was assumed to have been attained. At this point the RMS 

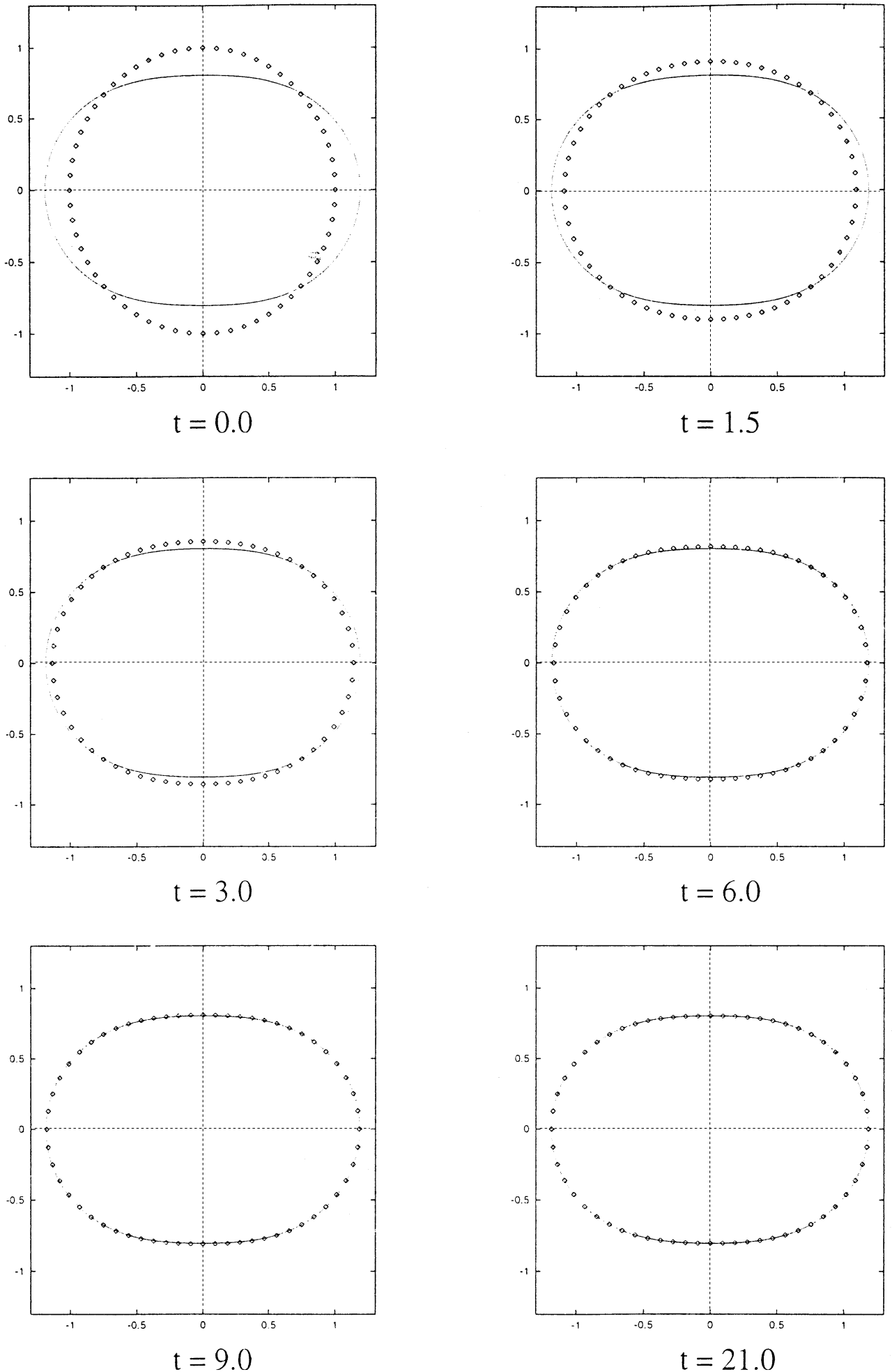

Figure 5. Evolution of blob to steady shape for $Q / \gamma D=1.667$. The continuous curve is the steady solution. 


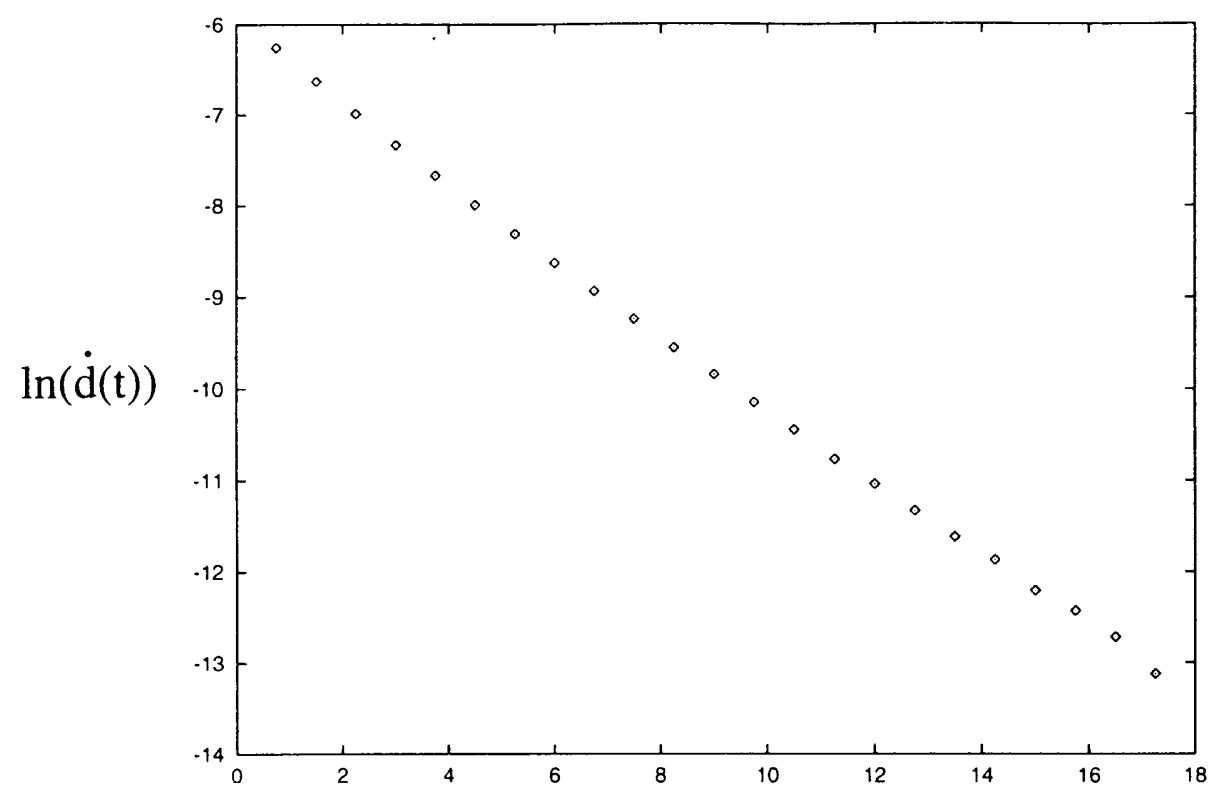

FIGURE 6. Drop off in log of speed of point at top of blob. $Q / \gamma D=1.667$. Slope of graph $\approx-0.4$.

error of the numerical to the theoretical solution was found to be $4.50 \times 10^{-5}$. The test was repeated, for $N=60$ and $N=80$. The results are displayed in Table 1 .

As a consequence of these results, convergence of the numerical solution to the analytic was assumed. The approach of the the shape to equilibrium for the $N=60$ case is illustrated in Figs. 5 and 6. Figure 5 displays the progress of the blob from an initial circle to its stable state. The analytic solution is shown also for comparison. Note that irregular timesteps are chosen, as most of the deformation takes place soon after switching on the flow. Figure 6 is a plot of $\ln \dot{d}(t)$ against time, where, as mentioned in $\S 4,(0, d(t))$ is the point where the interface cuts the positive $y$-axis. Note the exponential dependence of the velocity on time. The slope of the graph gives the exponent as $\approx-0.4$.

The test was repeated for various values of $Q / \gamma D$. For subcritical values, $Q / \gamma D<2.742$, the numerical solution converged similarly to the $Q / \gamma D=1.667$ case above to those steady shapes as given by the lower branch in Fig. 2. For $1.933 \lesssim Q / \gamma D<2.742$, the steady solutions were dogbone in shape, being more deformed for larger values of the flow strength. On taking a supercritical value of $Q / \gamma D,>2.742$, in this case $Q / \gamma D=3.0$, the blob was found not to converge to a steady shape, but to continue to deform, and eventually break by pinching off as the nodes lying on the $y$-axis met. Figure 7 displays the deformation of the blob for $Q / \gamma D=3.0$ as breakup approaches. Plots of the height, $d(t)$, and speed, $\dot{d}(t)$, of the point on the positive $y$-axis are given in Fig. 8. Note in Fig. 8 how $d(t)$ initially drops rapidly, then levels out somewhat, before dropping once again as the blob deforms towards breakup. This can also be seen from Fig. 7, as the shape remains roughly the same for $t=3$ to $t=12$.

As illustrated by the deformation curve, the theory of Entov et al. (1993) predicts either two or zero steady shapes for every value of $Q / \gamma D$ (ignoring the critical value). Shapes lying on the lower branch have been shown to be stable. Starting with a unit circle the 

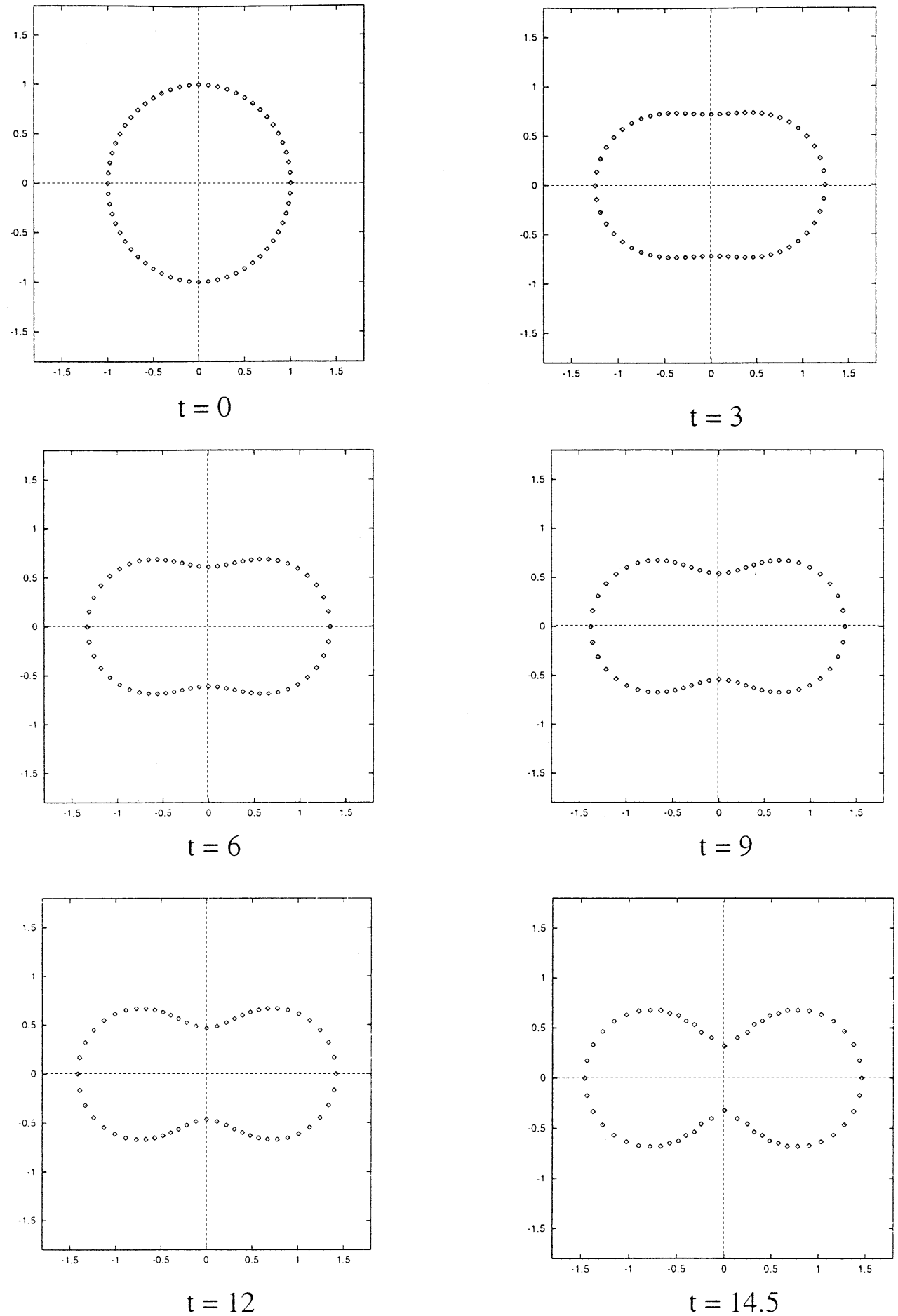

FIGURE 7. Time development of the blob for $Q / \gamma D=3.0$. Final picture is just before breakup occurs. 
Hele-Shaw quadrupole flow
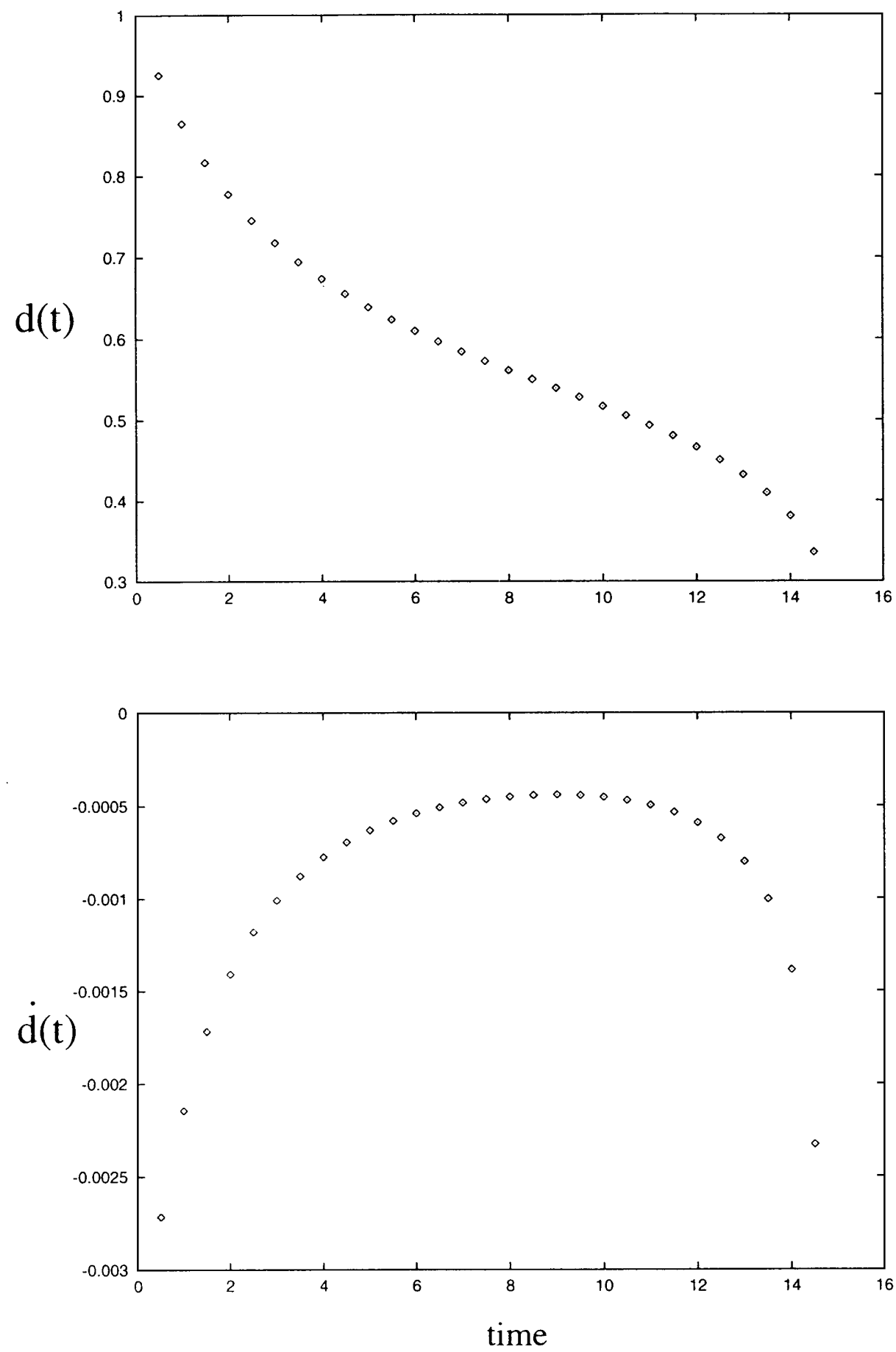

FiguRE 8 . Behaviour of point on positive $y$-axis for blob breakup. $Q / \gamma D=3.0$. 

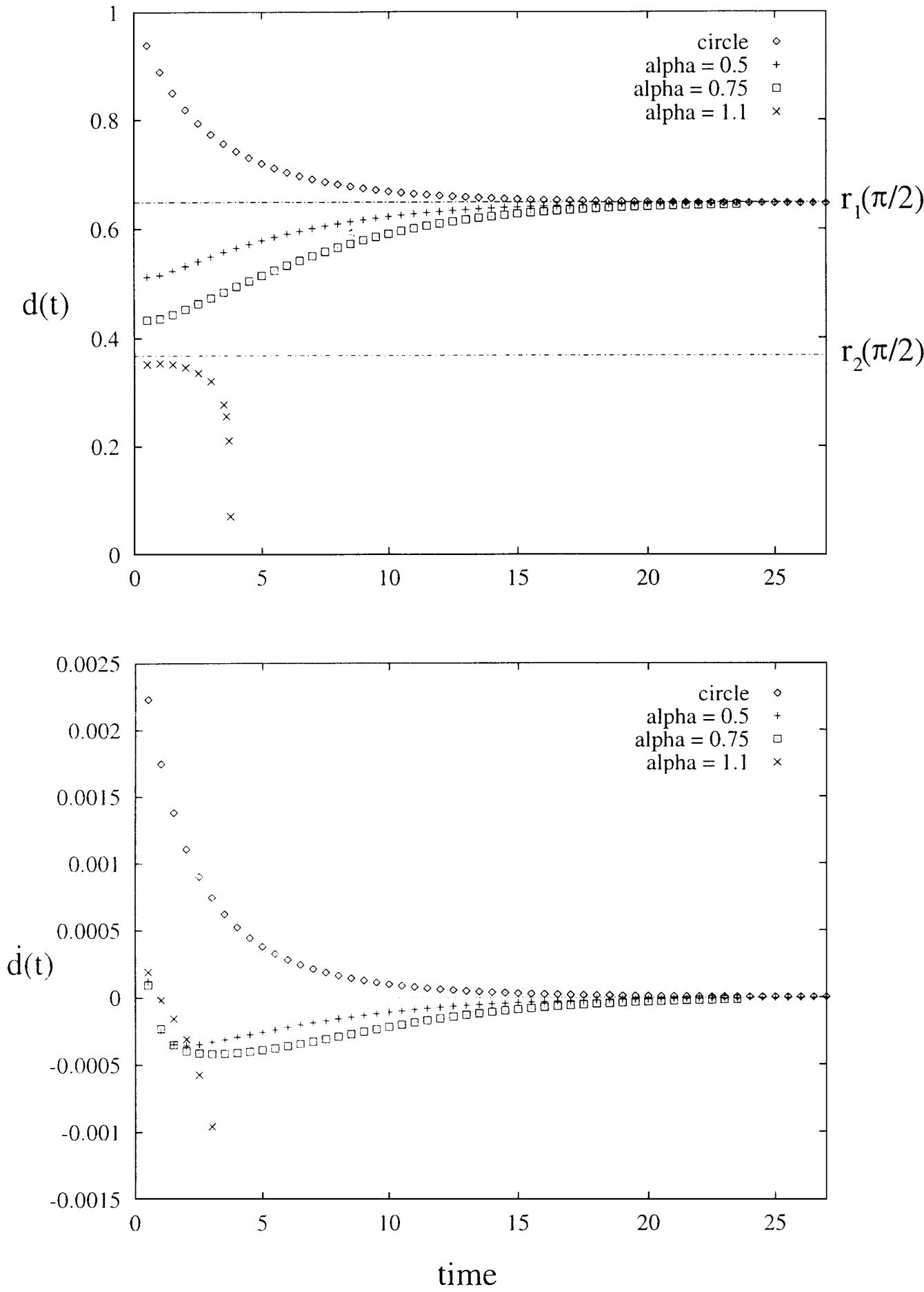

FIGURE 9. Behaviour of point on positive $y$-axis for $\alpha=0.5,0.75$ and 1.1 , and also initially circular state. $Q / \gamma D=2.5$. 
drop deforms to the steady shape, and remains there. However, we wish also to investigate the stability of those shapes lying on the upper branch. As these are unreachable from a circular initial configuration due to the stability of the lower branch, we chose to start with some shape in their neighbourhood as the initial configuration, a shape intermediate between those lying on the deformation curve for some suitable value of $Q / \gamma D$, and then see how this shape deforms to its final state once the flow is turned on. This is done via a linear superposition of the two known steady states for the particular $Q / \gamma D$ constructed as follows.

We let $\theta$ be the polar angle, and $r_{1}(\theta)$ be a mapping from the unit circle to the steady shape lying on the lower branch of the curve. Similarly, $r_{2}(\theta)$ is a mapping from the unit circle to the steady shape lying on the upper branch. The intermediate shape is then given by:

$$
r_{\alpha}(\theta)=(1-\alpha) r_{1}(\theta)+\alpha r_{2}(\theta),
$$

where $\alpha$ a positive real number and $r_{\alpha}(\theta)$ the resulting intermediate shape. Area, in general, is not conserved under this superposition. Therefore it is necessary to rescale $r_{\alpha}(\theta)$ to area $=\pi$ after construction.

For the purpose of this test $Q / \gamma D$ was taken to be 2.5. As can be ascertained from inspection of the deformation curve, both the shape from the lower branch and the upper are non-convex. For various trial values of $\alpha$, the intermediate shape, $r_{\alpha}(\theta)$ was calculated, given as the initial configuration and the flow then switched on. For $\alpha=0.5$ and 0.75 the blob eventually relaxed to the steady shape lying on the lower branch, $r_{1}(\theta)$. For $\alpha=1.1$ the blob broke as before. Fig. 9 displays the height, $d(t)$, and speed, $\dot{d}(t)$, of the point on the positive $y$-axis against time for each initial configuration. The results for starting from a circle initially are also displayed for comparison purposes. The plots for $\alpha=1.1$ are only continued out to $t=3.0$ as the blob breaks soon after the flow is switched on.

\section{Conclusions}

From the order of the error involved in predicting the steady shapes of Entov et al. (1993), and from the convergence of the solution with increasing number of nodes, we are confident that a code has been developed which accurately predicts the time-dependent solution for quadrupole driven flow in a Hele-Shaw cell with surface tension. During the course of this code development, the deformation curve of Figure 2 which predicts the steady shapes, if any, for a given value of $Q / \gamma D$ was produced. This deformation curve was derived from the mapping functions for the steady shapes found in Entov et al., but is not mentioned itself in that paper. The stability of these shapes has also been investigated, and it has been shown that shapes on the lower branch of the curve are stable, whereas those lying on the upper branch are unstable. There also exist values of $Q / \gamma D(\gtrsim 2.742)$ for which the strength of the quadrupole is too strong for surface tension forces to balance and no steady state exists. In this flow régime an initially circular blob of fluid will deform without reaching equilibrium, eventually breaking by pinching off along the $y$-axis. 


\section{Acknowledgement}

The computations were supported [in part] by the SERC 'Computational Science Initiative' Grant GR/H57585.

\section{References}

[1] DaI, W.-S. \& Shelley, M. J. (1993) A numerical study of the effect of surface tension and noise on an expanding Hele-Shaw bubble. Phys. Fluids A, 5(9), 2131-2146.

[2] Darcy, H. (1856) Les fontaines publiques de ville de Dijon. p. 560.

[3] Degregoria, A. J. \& Schwartz, L. W. (1986) A boundary-integral method for two-phase displacement in Hele-Shaw cells. J. Fluid Mech., 164, 383-400.

[4] Entov, V. M., Etingoff, P. I. \& Kleinbock, D. YA. (1993) Hele-Shaw flows with a free boundary produced by multipoles. Euro. J. Applied Maths., 4, 97-120.

[5] Hele-Shaw, H. J. S. (1898) The flow of water. Nature (London), 58, 34.

[6] Kadanoff, L. P. (1990) Exact solutions of Saffman-Taylor problem with surface tension. Phys. Rev. Lett., 65, 2986-2988.

[7] Lamb, H. (1932) Hydrodynamics. Cambridge University Press.

[8] Langer, J. S. (1980) Instabilities and pattern formation in crystal growth. Rev. Mod. Phys., 52, $1-28$.

[9] Meiburg, E. \& Homsy, G. M. (1988) Nonlinear unstable viscous fingers in Hele-Shaw flows. II. Numerical simulation. Phys. Fluids, 31, 429-439.

[10] Overman, E. A., Zabusky, N. J. \& Ossakow, S. L. (1983) Ionospheric plasma cloud dynamics via regularized contour dynamics. I. Stability and nonlinear evolution of one-contour models. Phys. Fluids, 26, 1139-1153.

[11] Park, C.-W. \& Homsy, G. M. (1984) Two-phase displacement in Hele-Shaw cells: theory. J. Fluid Mech., 139, 291-308.

[12] Pollard, D. D., Muller, O. H. \& Dockstader, D. R. (1975) The form and growth of fingered sheet intrusions. Geol. Soc. Am. Bull. 86, 351-363.

[13] Polubarinova-Kochina, P. Ya. (1945) On the motion of the oil contour. Doklay Akad. Nauk. SSSR, 47(4), 254-257.

[14] PozRikidis, C. (1992) Boundary Integral and Singularity Methods for Linearized Viscous Flow. Cambridge University Press.

[15] Saffman, P. G. \& TAYloR, G. I. (1958) The penetration of a fluid into porous medium or Hele-Shaw cell containing a more viscous liquid. Proc. R. Soc. A, 245, 312-329.

[16] Tryggvason, G. \& Aref, H. (1983) Numerical experiments on Hele-Shaw flow with a sharp interface. J. Fluid Mech., 136, 1-30

[17] Vasconcelos, G. L. \& Kadanoff, L. P. (1991) Stationary solutions for the Saffman-Taylor problem with surface tension. Phys. Rev. A, 44(10), 6490-6495. 